\title{
Some Unexpected Results of Experimental Measurement of Papers in Experiments in Fluids
}

\author{
Tang JH* and Wan DL
}

Departmental of Civil Engineering, Chung Yuan Christian University, Taiwan

Text

\section{ISSN: 2639-0574}

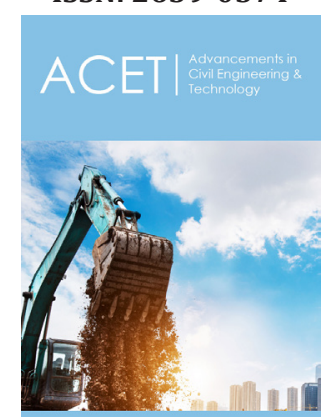

${ }^{* 1}$ Corresponding author: Tang JH, Departmental of Civil Engineering, Chung Yuan Christian University, Taiwan

Submission: 価 December 12, 2019

Published: 眥 December 16, 2019

\section{Volume 3 - Issue 5}

How to cite this article: Tang J, Wan D. Some Unexpected Results of Experimental Measurement of Papers in Experiments in Fluids. Adv Civil Eng Tech. 3(5). ACET.000574.2019.

DOI: 10.31031/ACET.2019.03.000574

Copyright@ Tang JH, This article is distributed under the terms of the Creative Commons Attribution 4.0 International License, which permits unrestricted use and redistribution provided that the original author and source are credited.
In this letter, the papers by Lin \& Sheu [1] and Wang et al. [2] were published by Experiments in Fluids have been carefully examined. A physically unreasonable phenomenon is that, based on the exact same experimental setups, for the parallel jets in paper by Lin \& Sheu [1], the locations of Merging Point (M.P.) and Combining Point (C.P.) are closer to the inlet section $(x=0)$ than those of inclined jets in paper by Wang et al. [2]. The figures reproduced from those two papers of experimental measurements are shown in the following Figure 1-4. In these figures, the space of the twin jets is $S=60 \mathrm{~mm}$, and the width of the jet is $D=2 \mathrm{~mm}$, thus $\mathrm{S} / \mathrm{D}=30$.

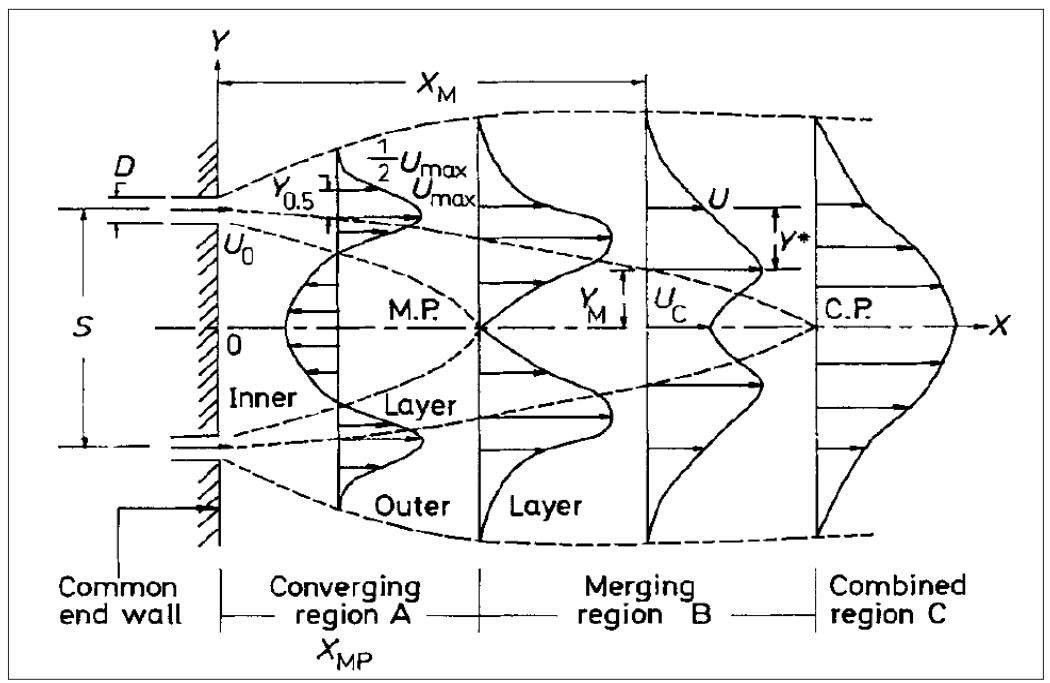

Figure 1: The notion defined in paper by Lin \& Sheu [1] for parallel jets.

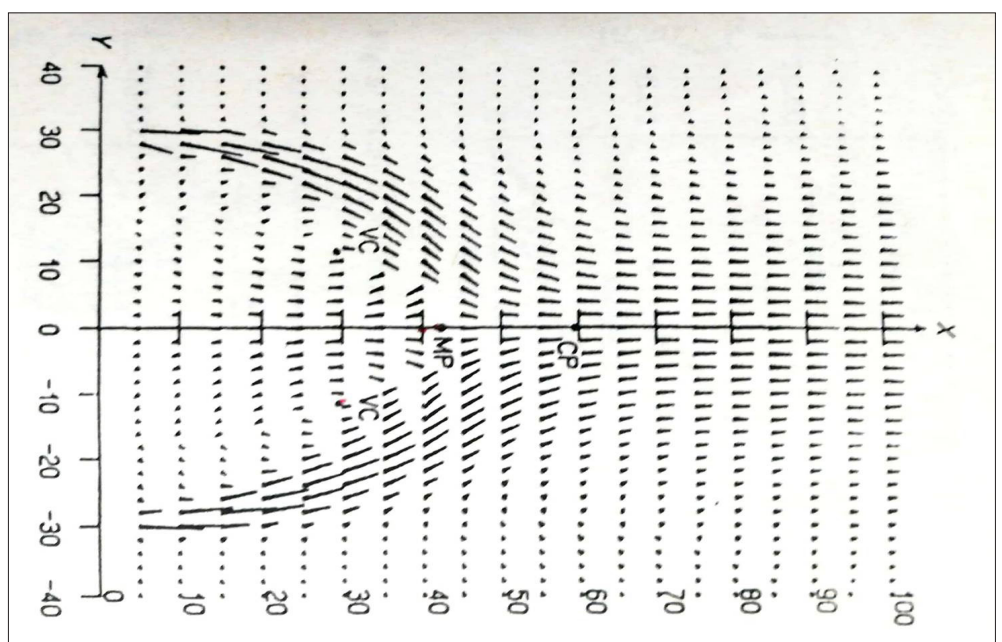

Figure 2: The location of M.P. and C.P. shown in the velocity distribution, from Figure 3 in paper by Lin \& Sheu [1]. 


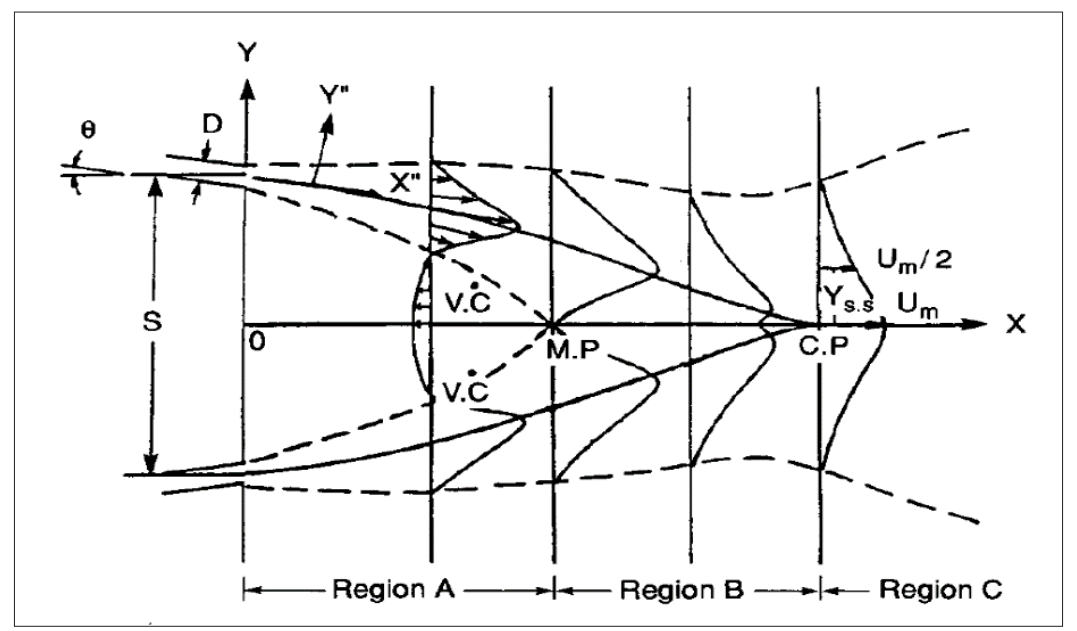

Figure 3: The notion defined in paper by Wang et al. [2] for inclined jets.

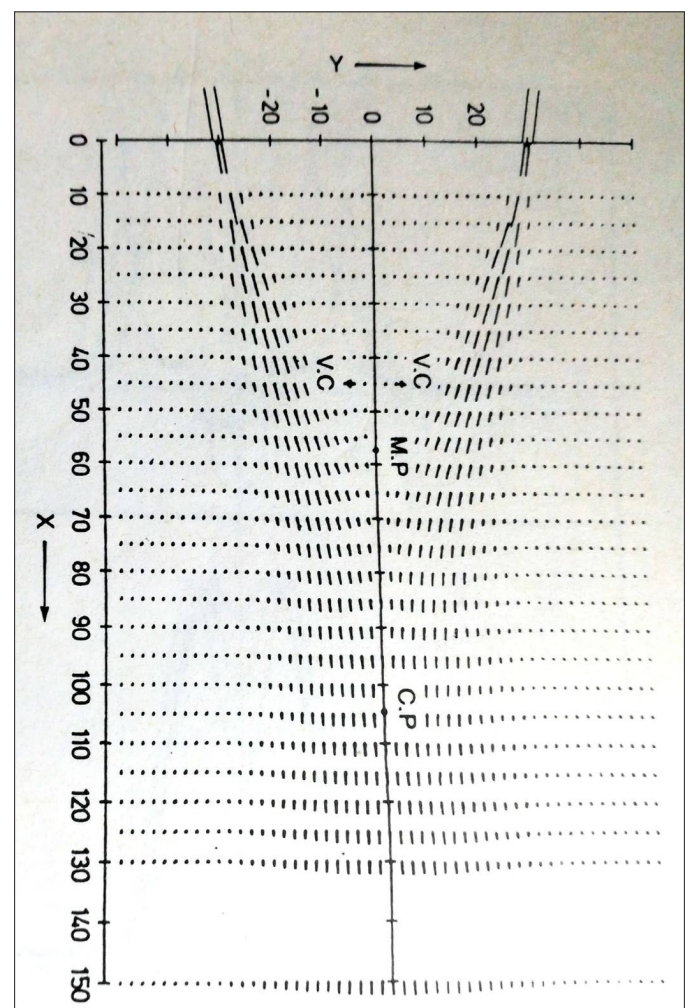

Figure 4: The location of M.P. and C.P. shown in the velocity distribution, from Figure 3 of paper by Wang et al. [2]. (For inclined angle, $\theta=9^{\circ}$ )

According to the experimental results, the positions of vortex center (V.C.), merging point, and combining point of both parallel and inclined dual jets are listed in Table 1. It's interesting to find out that for the same experimental setup for inflow $\left(Q=10 \mathrm{~m}^{3} / \mathrm{min}\right)$ and domain conditions, the positions, M.P. and C.P., of parallel dualjet flow are closer to the inlet section $(x=0)$ than those of inclined dual-jet flow. This result seems unreasonable and may need to be explained by the authors.

Table 1: Experimental measurements of V.C., M.P., and C.P. for S/D=30 with different inclined angels.

\begin{tabular}{|c|c|c|c|c|}
\hline \multirow{2}{*}{ Inclined Angel } & \multicolumn{2}{|c|}{ (Vortex Center, V.C.) } & (Merging Point, M.P.) & (Combining Point, C.P.) \\
\cline { 2 - 5 } & X/D & Y/D & X/D & X/D \\
\hline$\left(\theta=0^{\circ}\right)$ Lin \& Sheu [1] & 15 & 6 & 22.5 & 30.5 \\
\hline$\left(\theta=9^{\circ}\right)$ Wang et al. $[2]$ & 22.5 & 0.133 & 28.75 & 52.5 \\
\hline
\end{tabular}


In this article, according to the experimental setup, the numerical simulation was carried out by the commercial code
ANSYS Fluent [3]. The numerical simulation domain for parallel jets is shown in Figure 5.

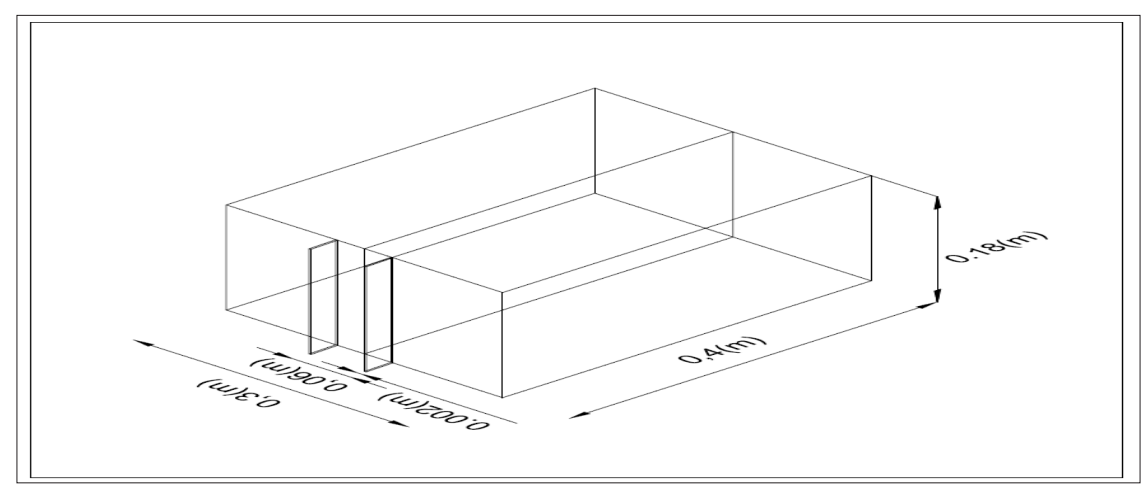

Figure 5: Domain of parallel jets for numerical simulation [3].

The comparison of numerical simulation and experimental the numerical simulation for the parallel jets, the cases of inclined measurements for parallel jets is shown in Table 2. For verification, jets of $9^{\circ}, 18^{\circ}$ and $27^{\circ}$ are set up for further simulations. The it's found that the simulation results are closed to the experimental comparison of results for experiment and simulation are shown in measurements in a reasonable discrepancy. After the verification of Table 3.

Table 2: Comparison of numerical simulation with the experimental measurements of the Vortex center, merging point, and Combining point for parallel jets, $\mathrm{S} / \mathrm{D}=30$.

\begin{tabular}{|c|c|c|c|c|}
\hline \multirow{2}{*}{$\begin{array}{c}\text { Angel } \\
\left(\boldsymbol{\theta}_{\mathbf{0}} \mathbf{}^{\mathbf{*}}\right.\end{array}$} & \multicolumn{2}{|c|}{ (Vortex Center, V.C.) } & (Merging Point, M.P.) & (Combining Point, C.P.) \\
\cline { 2 - 5 } & X/D & Y/D & X/D & X/D \\
\hline Numerical simulation by Wan [3] & 12 & 6.1 & 23.75 & 31.25 \\
\hline Experimental measurement by Lin \& Sheu [1] & 15 & 6 & 22.5 & 30.5 \\
\hline
\end{tabular}

Table 3: For dual-jet flow with inclined inlet jets, V.C., M.P., and C.P. for S/D=30 of numerical simulation [3] and experimental measurement [2].

\begin{tabular}{|c|c|c|c|c|c|}
\hline \multicolumn{2}{|c|}{$\begin{array}{c}\text { Angel } \\
(\boldsymbol{\theta})\end{array}$} & \multicolumn{2}{c|}{$\begin{array}{c}\text { (Vortex Center, V.C.) } \\
\text { (Merging Point, M.P.) }\end{array}$} & (Combining Point, C.P.) \\
\cline { 2 - 6 } \multicolumn{2}{|c|}{\begin{tabular}{c} 
X/D \\
\cline { 2 - 5 }
\end{tabular}} & Y/D & X/D & X/D \\
\hline \multirow{2}{*}{$9^{\circ}$} & Experiment measurement [2] & 22.5 & 0.133 & 28.75 & 52.5 \\
\hline \multirow{2}{*}{$18^{\circ}$} & Numerical simulation [3] & 13.1 & 5.9 & 21.5 & 30.5 \\
\cline { 2 - 6 } & Experiment measurement [2] & 20 & 0.12 & 26.25 & 45 \\
\hline \multirow{2}{*}{$27^{\circ}$} & Numerical simulation [3] & 10.6 & 4.45 & 18.75 & 25 \\
\cline { 2 - 6 } & Experiment measurement [2] & 14 & 0.1 & 20.05 & 30 \\
\hline
\end{tabular}

In Table 3, the distance between both M.P. and C.P. to the inlet section $(x=0)$ is reducing with increasing inclined angels for both experimental and numerical results. The numerical simulation of M.P. and C.P. is reasonable for comparing with the case of parallel jets. However, for the experimental values of M.P. and C.P. for inclined angel $9^{\circ}$ and $18^{\circ}$ are still larger than the value of parallel jets. Therefore, there could exist some errors need to be explained in the paper by Wang et al. [2].

\section{References}

1. Lin YF, Sheu MJ (1990) Investigation of two plane parallel unventilated jets. Exp Fluids 10(1): 17-22.

2. Wang CS, Lin YF, Shue MJ (1993) Measurements of turbulent inclined plane dual jets. Exp Fluids 16(1): 27-35.

3. Wan DL (2018) Numerical study on the flow and concentration fields in two plane unventilated jets. Master thesis, Chung Yuan Christian University, Taiwan.

For possible submissions Click below: 